\section{Estudo seccional descritivo de crianças com deficiência auditiva atendidas no Instituto Nacional de Educação de Surdos, Rio de Janeiro, Brasil}

\author{
Descriptive cross-sectional study of \\ hearing-disabled children at the \\ National Institute for Education of \\ the Deaf in Rio de Janeiro, Brazil
}

Eduardo Jorge Custódio da Silva

Juan Clinton Llerena Jr. 1

Maria Helena Cabral de Almeida Cardoso 1

\footnotetext{
I Instituto Fernandes Figueira, Fundação Osvaldo Cruz, Rio de Janeiro, Brasil.

Correspondência E. J. C. Silva Instituto Fernandes Figueira, Fundação Osvaldo Cruz. Rua Eduardo Guinle 55, Bloco 2, apto. 204, Rio de Janeiro, $R J$ 2220-090, Brasil. eduardo.jorge@openlink.com.br
}

\begin{abstract}
In Brazil, research is scarce on multiple disabilities and hearing disability in particular. Researchers began a pioneering study in 1992, evaluating students from public special education programs, currently focused on hearing disability. The authors evaluated 232 students ranging from 1 to 39 years of age (mean 10.9 years), with male gender prevailing. Consanguinity was present in $7.6 \%$ and a family history of deafness in 19\%. Gestational complications were recorded in 33\% of cases. Normal labor occurred in $72 \%$, and delivery at term in $75 \%$. Neonatal complications were present in $35 \%$ of the sample. Environmental causes accounted for 56\% of the sample, genetic causes $20.7 \%$, and the remaining $20.7 \%$ were classified as idiopathic. The current findings corroborate those from the literature. We believe that this study can spark greater concern for the hearing-disabled and that through increased knowledge of this group's characteristics it may foster strategies to facilitate interaction with society as a whole.
\end{abstract}

Hearing Impaired Persons; Cross-Sectional Studies; Special Education

\section{Introdução}

Segundo a Organização Mundial da Saúde (OMS) 1 existem mais de 120 milhões de pessoas no mundo com perda auditiva, sendo que 8,7 milhões têm idade variando de 0 a 19 anos, mostrando que crianças nascem surdas ou têm perda auditiva quando muito jovens. Os dados atuais apontam que seis em cada mil crianças apresentam déficit auditivo ao nascimento e que uma em cada mil tornam-se deficientes auditivas antes da idade adulta ${ }^{2}$. Por exemplo, somente nos Estados Unidos da América, a Hearing Loss Organization refere que a perda de audição, desde aquela afetando a compreensão da fala até a surdez total, afeta 28 milhões de americanos, esperando-se que por volta de 2030 este número esteja duplicado 2 . Fatores genéticos são considerados como a causa de mais de $60 \%$ dos casos de surdez congênita ou precoce equivalentes às formas não sindrômicas, e na maioria das vezes estão associados a uma única mutação gênica ${ }^{3}$. Cerca de $75 \%$ dos indivíduos com surdez geneticamente determinada não apresentam nenhuma outra característica clínica, e os restantes $25 \%$ têm síndromes reconhecidas ${ }^{4}$. Estima-se que cerca de $75 \%$ dos casos são de herança autossômica recessiva, $15 \%$ dos casos de herança autossômica dominante, 2 a 3\% dos casos de herança ligada ao cromossomo $\mathrm{X}$ e, ainda, nessa mesma última proporção encontram-se os casos de herança mitocondrial 4 . 
De um modo geral classifica-se a perda de audição por condições genéticas como sindrômica e não sindrômica. Estima-se que há cerca de 30 a 100 genes para a perda auditiva não sindrômica autossômica recessiva. Por volta de 60 loci já foram localizados (32 loci autossômicos dominantes, 28 loci autossômicos recessivos e 5 loci ligados ao X) 5 .

Como a audição é um dos principais canais de informação do homem deve-se contemplá-la como um fator de vital importância para a qualidade de vida das populações. Sendo uma das principais responsáveis pela aquisição da linguagem, processo que envolve desenvolvimento de pensamento, memória e raciocínio, a perda auditiva presente desde o nascimento ou estabelecida na mais tenra infância irá interferir significativamente no processo de desenvolvimento da criança. Por outro lado, a surdez numa pessoa adulta leva a uma sensação de isolamento, podendo torná-la dissociada de sua comunidade. É fácil perceber que é passível também de fazê-la mais vulnerável a ameaças externas, cerceando sua capacidade de atuar com independência e autonomia. Prevenir se torna então o caminho a ser construído e compartilhado pelos profissionais de saúde e/ou educação que têm em mente a saúde física e psíquica dos indivíduos, assim como sua inserção eficaz e profícua na vida societária.

Pretendemos com a discussão empreendida nesse trabalho, cujo objeto é a descrição das características clínicas e epidemiológicas de uma população inserida em um programa de educação especial de referencia nacional, chamar a atenção para a necessidade de se juntar esforços para elaborar ações eficazes à detecção precoce da perda auditiva.

\section{Materiais e métodos}

Este trabalho integra uma linha de pesquisa que vem sendo desenvolvida no Instituto Fernandes Figueira, Fundação Oswaldo Cruz (IFF/ FIOCRUZ), no Departamento de Genética Médica. Desde 1992 esta linha do Diretório de Pesquisa do Conselho Nacional de Desenvolvimento Científico e Tecnológico - CNPq (Centro de Genética Médica José Carlos Cabral de Almeida) avalia os transtornos do desenvolvimento em crianças usuárias de vários tipos de atendimento pedagógico existentes no Estado do Rio de Janeiro, Brasil. Até o final de 2002 foi avaliado um total de 1.500 crianças que apresentavam algum tipo de distúrbio do desenvolvimento, tendo-se registrado a surdez em 160 delas.

A partir de 2003 deu-se início à avaliação dos alunos do Instituto Nacional de Educação de Surdos (INES), centro de referência nacional no atendimento à surdez. Foram examinados, até 2005, 232 alunos de um total de 500, escolhidos aleatoriamente em todos os segmentos do INES. As entrevistas foram feitas com seus responsáveis, seguindo dois protocolos clínicos de investigação, um genético e o outro neurológico, já validados e utilizados nas etapas anteriores 6,7,8. Contudo, especificamente para esta etapa foi acoplado o registro dos métodos de diagnósticos da surdez, da época em que tal diagnóstico foi comprovado e do início da ação terapêutica. A incorporação dessas variáveis respondeu pela lacuna detectada a partir da análise das entrevistas previamente realizadas com os pais e responsáveis pelos alunos surdos das escolas estaduais, da Secretaria Municipal e daqueles inseridos no modelo pedagógico da Fundação Municipal de Niterói, realizadas de 1992 a 2000.

As avaliações no INES foram empreendidas por uma equipe composta por geneticista clínico, neurologista e assistente social, com baixo índice de absenteísmo por parte dos alunos. Representantes da instituição e professores participaram do trabalho. A avaliação neurológica foi feita pelo mesmo investigador em todos os casos, consistindo em exame neurológico clássico e no exame neurológico evolutivo ${ }^{9}$. O questionário genético e o exame morfológico foram aplicados e realizados por mais de um pesquisador, entretanto todos tiveram o mesmo treinamento em serviço, supervisionados pelo pesquisador senior. Tanto o questionário, re-validado após a inclusão das variáveis acima referidas, quanto o exame morfológico, se basearam em conceitos e orientações preconizadas por Jones 10, o que ensejou a homogeneidade da coleta. A presença de um responsável do aluno foi sempre exigida para autorização do exame clínico e assinatura do Termo de Consentimento Livre e Esclarecido, sendo a não concordância com o mesmo o único critério de exclusão.

Diante da complexidade de estabelecer diagnósticos etiológicos numa população tão heterogênea quanto a composta por deficientes auditivos, e cientes das limitações existentes nesse tipo de investigação exploratória, seccional e retrospectiva, classificamos nossa amostra, compreendendo um total de 232 indivíduos, em três grandes grupos etiológicos: genético, ambiental e idiopático. Foram categorizados como pertencentes ao grupo genético os alunos que apresentavam um conjunto de dismorfias faciais peculiares associadas ou não a outras malformações congênitas, doenças genéticas de diagnóstico reconhecidamente baseado em critérios clínicos (neurofibromatose tipo II, Usher, Waardemburg, entre outras), consangüinidade parental na au- 
sência de fatores ambientais adversos associados, dois indivíduos do sexo masculino afetados, ou dois indivíduos com dismorfias faciais peculiares similares, ou ainda apresentando a deficiência em questão em mais de duas gerações. Foi utilizado, também, em poucas ocasiões, o relatório médico do aluno com o diagnóstico etiológico definitivo. No grupo ambiental foram inseridos os indivíduos com história patológica pregressa de eventos externos, não constitucionais, identificados pelos questionários, como, por exemplo, infecção congênita, descolamento prematuro da placenta, prematuridade, asfixia perinatal, tocotraumatismo, uso de antibióticos ototóxicos, meningoencefalites, traumatismo craniano, dentre outros. No grupo idiopático foram incluídos os alunos cujos questionários e exame físico não foram capazes de identificar fatores genéticos e ou ambientais como causas do déficit auditivo.

As informações clínicas coletadas em campo constituíram um banco de dados e foram analisadas através dos testes estatísticos do Programa Epitable e do Programa Statcalc integrantes do pacote de programas Epi Info 6.0 (Centers for Disease Control and Prevention, Atlanta, Estados Unidos).

Os dados referentes à população geral brasileira no que tange ao sexo, tipo de parto, idade materna ao nascimento do concepto e consangüinidade parental, para fins de comparação com os achados da pesquisa, foram obtidos a partir de duas fontes: pela declaração de nascidos vivos de 2002 do Município de Niterói, Rio de Janeiro, Brasil, e de duas maternidades do grupo Estudio Colaborativo Latino-Americano de Malformaciones Congenitas (ECLAMC), em dois períodos distintos, de 1982 a 1990 e de 1988 a 1994, num total de 18.337 registros 11.

Os dados coletados foram monitorados para garantir a uniformidade das avaliações, mediante a realização de reuniões periódicas com a participação de todos os envolvidos. Nessas ocasiões sistematicamente todos os questionários eram revisados para se ter certeza do preenchimento de todas as questões formuladas, assim como se discutiam casos individuais de interesse geral ou cuja avaliação havia suscitado questionamentos ou levantado hipóteses diagnósticas por parte de qualquer membro da equipe. Encerrada a discussão, processava-se o armazenamento dos dados. Um relatório médico contendo o diagnóstico etiológico da surdez, riscos de recorrência familiar, segundo as leis clássicas mendelianas de segregação, e condutas pedagógicas, era confeccionado e encaminhado para cada família entrevistada.

\section{Resultados}

Foram avaliados 232 surdos. A média de idade foi de 10,9 anos, entretanto, para fins estatísticos, depois de selecionadas as variáveis a serem analisadas, só se computou o número de casos que possuíam a informação necessária.

Com relação ao sexo observamos o predomínio do masculino (65\%), o que nos aproxima dos achados relatados por Marazita et al. 4 (52,33\%), sendo que estes dados apresentam relevância estatística (Tabela 1).

Tabela 1

Características e antecedentes em 232 alunos com surdez no Instituto Nacional de Educação de Surdos (INES).

\section{Características e antecedentes}

\begin{tabular}{lc}
\hline Faixa etária (em anos) & $1-39$ (média 10,9) \\
Sexo (m:f) & $1,55: 1,00$ \\
História familiar de surdez (\%) & 19,0 \\
Consangüinidade (\%) & 7,6 \\
Idade diagnóstico da surdez (anos) & 3,2 \\
Tipos de parto (\%) * & \\
Normal hospitalar & 57,00 \\
Normal domiciliar & 1,33 \\
Cesáreo & 40,90 \\
\hline
\end{tabular}

* Declaração de nascidos vivos (Município de Niterói, Rio de Janeiro, Brasil, 2002).

O quadro clínico mais evidente é o mesmo reportado pela literatura especializada, ou seja, encontrado em pessoas com perda maior que $55 \mathrm{~dB}$ (perda acentuada). Contudo, a literatura também acentua que pacientes com perda leve ou moderada podem apresentar dificuldades para ouvir de forma plena as mensagens, o que leva ao aparecimento de problemas no desenvolvimento das potencialidades de comunicar-se, de desempenho acadêmico e de funcionamento social.

Na maior parte das vezes, conforme a revisão bibliográfica apontou, a identificação dos casos foi feita pela própria família e relatada ao profissional de saúde, que habitualmente não valoriza a queixa. Essa talvez seja uma das razões de a média de idade do diagnóstico da deficiência auditiva grave à profunda seja de aproximadamente dois anos, com atraso de um ano entre a suspeita dos pais e a referência do pediatra ao profissional especializado.

Encontramos a presença de uniões consangüíneas em 16 casos (7,6\%), fato já relatado por 
Ucherman no ano de 1869, e que pode ser também justificado pelo grande número de uniões entre pessoas com deficiência auditiva, que chega a $90 \% 12$.

Quando a amostra foi classificada considerando a provável etiologia, encontramos o predomínio das causas ambientais $(58,5 \%)$, seguidas pelas idiopáticas $(20,7 \%)$ e genéticas $(20,7 \%)$. A importância das causas ambientais, portanto evitáveis, indica a necessidade de se melhorar o acesso aos serviços de saúde e as condições de vida de nossa população.

Outro fato evidenciado por nossa pesquisa, também discutido por Rodrigues \& Noronha 13, é a presença de familiares com surdez que, em nosso caso, foi de 43 alunos ou 15\% do total dos casos, configurando uma tradição de uniões conjugais dentro da comunidade de surdos.

A distribuição da idade materna ao nascimento por faixas etárias não foi muito diferente daquela observada na população geral. E a paterna pareceu seguir um padrão compatível com a distribuição anterior.

A análise das características gestacionais sugere condições precárias de atendimento pré-natal e de planejamento familiar, porque embora a maior parte da amostra situe-se até quatro partos e gestações, o número de mães que tiveram mais de quatro gestações $(20,1 \%)$ e de quatro partos $(18,1 \%)$ mostrou-se significativo.

Observamos a ocorrência de abortamentos, quer espontâneos ou não, em 19,1\% da amostra, sem significância estatística. Foi também encontrada história de intercorrências gestacionais em 23,3\% dos casos avaliados

Obtivemos uma taxa de partos normais de $59 \%$, tendo sido a maior parte deles realizada em ambiente hospitalar $(57,7 \%)$, dado relevante se comparado com os dados da declaração de nascidos vivos, da mesma forma que os $2 \%$ de partos domiciliares.

Com relação à época do parto, constatamos prematuridade em $21 \%$ da amostra, sendo que $2 \%$ dos alunos examinados eram nascidos póstermo, o que não configurou relevância estatística.

A média registrada de $2.928 \mathrm{~g}$ de peso ao nascimento, somente passível de ser documentada no caso de 197 alunos, sugere o baixo peso ao nascer como fator de risco, porém sem relevância estatística.

As intercorrências neonatais ocorreram em $33,3 \%$, sendo que grande maioria das mães referiu internação em berçário por prazos variados. A asfixia relatada pelas mães ocorreu em $6,1 \%$ (intervalo de confiança entre 3,01 e 6,11\%), quando comparada com os dados da declaração de nascidos vivos, apresentando relevância estatís- tica. A utilização de antibiótico $(3,4 \%)$, de fototerapia $(2,7 \%)$ e as convulsões $(1,4 \%)$, durante o período, também foram narradas, confirmando a importância das condições neonatais na gênese da surdez.

Outro aspecto relevante embutido em nossos resultados é a problemática das alterações comportamentais reportadas pelas mães, que foram observadas em 36 alunos, sendo a hiperatividade a queixa predominante. No entanto, é reconhecida a agitação psicomotora de crianças pequenas com surdez, principalmente, quando não há um bom canal de comunicação, fato que é a regra nesses alunos, pois como já foi apontado anteriormente, e ver-se-á a seguir, o diagnóstico e a intervenção são tardios.

O método diagnóstico reportado como o mais utilizado foi a audiometria de tronco (BERA), em $67,5 \%$ dos casos (Figura 1). Tal fato e praticamente a ausência de diagnóstico por otoemissão acústica podem ser explicados em função da idade média da amostra. Porém também deve ser ponderada a cultura médica que considera a audiometria de tronco como o único exame capaz de confirmar a surdez.

$\mathrm{Na}$ avaliação das prováveis etiologias, as infecções congênitas foram prevalentes, salientando-se a rubéola. Cabe ressaltar, todavia, que quase a metade dos casos avaliados foram diagnosticados por parâmetros clínicos, não comprovados por exame laboratorial. As meningoencefalites também se apresentaram como importante causa de surdez.

A Tabela 2 exemplifica, através de nossos resultados, a importância das causas ambientais e em conseqüência passíveis de sofrerem a ação de medidas preventivas primárias, tais como a vacinação em massa se atentando, mormente, para os números referentes à rubéola.

\section{Discussão}

Em estudo recente, Parving et al. 14, após reverem a perda de audição em crianças ao longo de trinta anos, na cidade de Copenhague, Dinamarca, reportaram que a média de idade do diagnóstico das crianças nascidas de 1990 a 1999 foi de 18 meses, de 1980 a 1989 foi de 16 meses e de 1970 a 1979 foi de 43 meses. Concluem que, já se aproximando da virada do século XX para o XXI, o diagnóstico precoce era raro, pois, o inquérito realizado apontou que antes dos seis meses só foram identificados $6 \%$ dos casos de deficiência auditiva congênita, e somente $27 \%$ foram estabelecidos aos 24 meses de vida.

Os dados referentes às causas ambientais auferidos por nossa pesquisa salientam ainda mais 
Figura 1

Método diagnóstico da surdez em 199 alunos matriculados no Instituto Nacional de Educação de Surdos (INES),

Rio de Janeiro, Brasil.

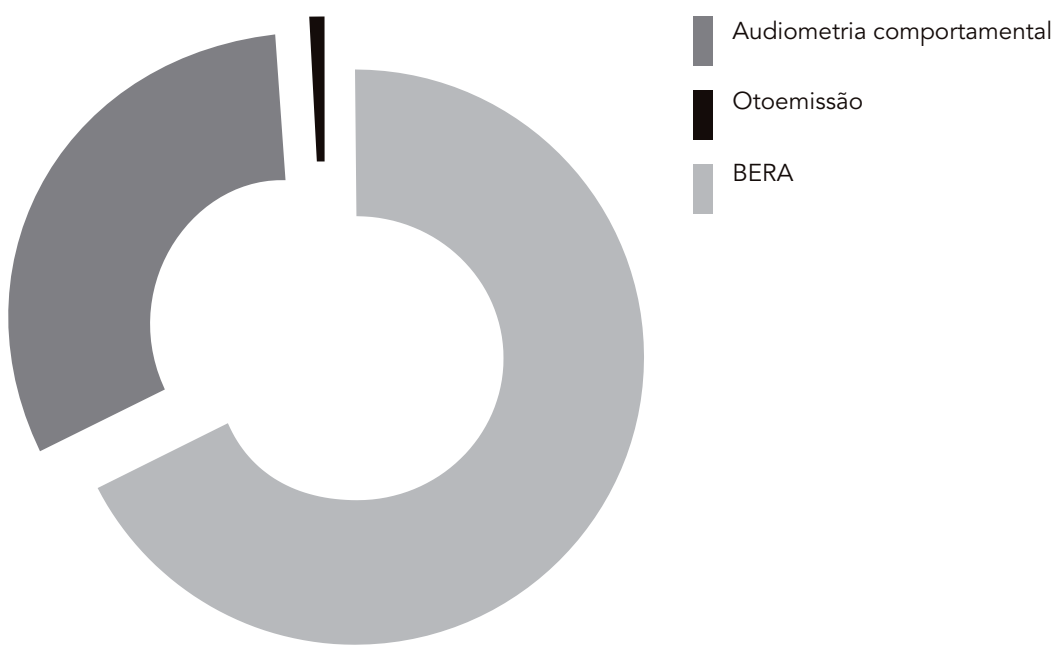

Tabela 2

Classificação clínico-etiológica de 232 alunos * com surdez em escolas de educação especial no Estado do Rio de Janeiro, Brasil.

\begin{tabular}{|c|c|c|c|c|c|}
\hline \multicolumn{3}{|c|}{ Genético ( $N=50 ; 20,70 \%)$} & \multicolumn{3}{|c|}{ Ambiental $(N=132 ; 56,00 \%) * \star$} \\
\hline Síndrome & $\mathrm{n}$ & $\%$ & Evento & $\mathrm{n}$ & $\%$ \\
\hline História familiar & 24 & 48,00 & Rubéola & 34 & 25,75 \\
\hline 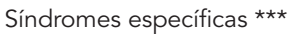 & 10 & 20,00 & Meningite & 28 & 21,20 \\
\hline Dismorfias faciais & 8 & 16,00 & Prematuridade & 20 & 15,10 \\
\hline Consangüinidade & 5 & 10,00 & Uso de antibiótico & 15 & 11,36 \\
\hline Waardenburg & 2 & 4,00 & Asfixia & 11 & 8,33 \\
\hline \multirow[t]{8}{*}{ Usher } & 1 & 2,00 & Otites & 7 & 5,30 \\
\hline & & & Hiperbilirrubinemia & 7 & 5,30 \\
\hline & & & Infecções congênitas & 4 & 3,00 \\
\hline & & & Traumatismo craniano & 2 & 1,50 \\
\hline & & & Síndrome hipóxico- & & \\
\hline & & & isquêmica pós-natal & 2 & 1,50 \\
\hline & & & Sarampo & 1 & 0,75 \\
\hline & & & Sífilis & 1 & 0,75 \\
\hline
\end{tabular}

* Cinqüenta alunos (20,70\%) foram considerados de causa idiopática;

** De acordo com informações colhidas;

*** Síndromes específicas = Saethre-Chotzen, Shprintzen, Treacher Collins, crânio-fronto-nasal, espectro óculo-aurículo-vertebral, associação com DandyWalker, Klipel-Feil, Johasson Blizard, neurofibromatose tipo II e Coffin-Lowry. 
a importância do diagnóstico e da intervenção precoce, o que, obviamente, tem impacto no prognóstico. Em função disso o Joint Comitee on Infant Hearing 15 elaborou uma lista de indicadores associados à perda auditiva para incentivar a identificação precoce das crianças com surdez. Esta lista compreende: construção da história familiar para perda auditiva sensório-neural hereditária; checagem de possível histórico de infecção intra-uterina atentando-se para citomegalovirus, rubéola, sífilis, herpes e toxoplasmose; investigação de anomalias crânio faciais, incluindo anormalidades morfológicas da pina e do canal auditivo; atenção para peso ao nascer inferior ao 1.500g; checagem de hiperbilirubinemia num nível seroso que requisitou exasanguíneo transfusão; história de meningite bacteriana; registro de Apgar de 0 a 4 no primeiro minuto ou 6 aos cinco minutos; uso de ventilação mecânica por cinco dias ou mais; sinais clínicos ou outros achados associados a síndromes que reconhecidamente incluem perda auditiva sensório-neural ou condutiva e, por fim, relato do uso de substâncias ototóxicas, incluindo os aminoglicosídeos.

No que tange às possíveis causas da surdez, o relatório anual de crianças e jovens deficientes auditivos de 1988 a 1989, recuperado por Marazita et al. 4, compreendendo 47.864 indivíduos, nos Estados Unidos, apresentava a seguinte distribuição: 37\% dos casos eram de causa ambiental (ou surdez adquirida), 49,5\% inseriam-se no grupo idiopático e 13,2\% dos casos tinham causa genética. Esses dados já chamavam a atenção para a tendência de diminuição do grupo de caráter ambiental, que em relatório prévio, datado de 1970, correspondia a 49,3\% dos casos estudados. Os dados referentes ao grupo ligado às causas desconhecidas, quando posteriormente analisados, através de técnicas estatísticas mais apuradas, revelaram possíveis fatores genéticos como determinantes do déficit auditivo. Deve-se ressaltar o fato de que apenas $30 \%$ dos pacientes, cuja surdez era geneticamente determinada, possuíam síndromes específicas, dificultando o diagnóstico em bases meramente clínicas, pois o restante não apresentava outra alteração que não a própria deficiência em questão. Pode-se, portanto, inferir que as recomendações do Joint Comitee on Infant Hearing, publicadas em 2002 15, acentuam a manutenção dos esforços que vêm sendo empreendidos, enfatizando, sobretudo, a importância do rastreamento neonatal.

Com o advento de novas propedêuticas médicas e a maior disponibilidade de serviços especializados, principalmente os concernentes às medicinas fetal e neonatal, assistimos, nos últimos anos, ao crescimento da população de risco para o desenvolvimento de doenças crônico-de- generativas, com a correspondente ampliação da expectativa de vida. Essa nova realidade vem sofrendo revisões tanto no que diz respeito às ações médicas quanto às suas implicações éticas 16,17, tecnobiocientíficas 18,19 e sócio-econômicas 20,21, sendo alvo de debates e discussões em todos os meios de troca e difusão, desde artigos em revistas especializadas a reuniões, simpósios, mesas redondas, congressos, entre outros, compreendendo as mais variadas áreas das chamadas ciências humanas e da vida 22,23 .

Os esforços têm cada vez mais se dirigido para tentar detectar as populações de risco, partindo-se de protocolos clínicos ou através de inventários de risco perinatal, empreendendo-se toda uma ação no sentido de intervir o mais precocemente possível 24,25.

No que tange à presença de familiares surdos, na árvore genealógica dos alunos componentes de nossa amostra, a explicação comumente encontrada é a de que entre os deficientes auditivos registra-se a tendência de agregarem-se como uma espécie de etnia, vivendo em comunidades específicas. Na verdade, o grupamento formado por deficientes auditivos representa para alguns sociólogos, antropólogos e lingüistas uma variação cultural dentro da própria sociedade 13 .

Diante de nossos resultados, acreditamos que a incidência de partos normais esteja superestimada, uma vez que temos dados relativos ao ano de 1996, isto é, existem, dentre aqueles que compuseram a amostra, alunos nascidos há mais de vinte anos, e sabidamente o número de partos cesáreos no Brasil vem aumentando 26.

Embora a prematuriade seja fator de risco reconhecido para a surdez tanto direta quanto principalmente indireta devido às complicações associadas (asfixia, uso de antibióticos, hemorragias periventriculares, dentre outras), o melhor desempenho médico nos cuidados aos prematuros, asfixiados, com anomalias e malformações congênitas, afetados por doenças genéticas entre outras, tem propiciado uma maior longevidade 27,28 , com melhoria na qualidade de vida. A maior difusão das UTIs neonatais e o avanço das tecnologias disponíveis têm aumentado a sobrevida dos recém-nascidos de peso cada vez menor, portanto, gradativamente e em número cada vez maior, crianças que anteriormente não tinham a perspectiva de nenhum tipo de treinamento, aperfeiçoamento ou inserção escolar, hoje, encontram meios de desenvolver suas potencialidades nas principais capitais brasileiras.

No Brasil, até o presente momento, os serviços de estimulação essencial estão organizados em creches, escolas especiais, organizações não governamentais e até no próprio ambiente doméstico, sempre contando com o apoio de equi- 
pes especializadas, porém, em sua maioria, sem notificação oficial ao Estado, denotando o que de muito tem sido feito, mas o que ainda de muito há para fazer.

Multiplicam-se os estudos de levantamento das populações de risco pré, peri e pós- natais, como a de prematuros, asfixiados, portadores de malformações variadas, pacientes com traumatismo crânio encefálico, vítimas de infecções do sistema nervoso central, dentre outras 29,30,31,32. Avaliações clínicas (inventários de risco) 33, de imagem (ultra-som transfontanela, tomografias, ressonâncias) 34,35, neurofisiológicas (eletroencefalogramas e potenciais evocados) 36 e laboratoriais (teste do pezinho, medidas de consumo de oxigênio) 37,38 são meios importantes na investigação de marcadores objetivos que podem auxiliar a diagnosticar eventuais seqüelas ocorridas, a fim de facilitar projetos eficazes de intervenção precoce. Ao revisar-se a literatura internacional especializada, observa-se o declínio das causas chamadas ambientais, justamente pela intervenção precoce ativa no sentido de prevenir essas patologias, por meio do uso de vacinas contra a rubéola e o Haemophilus, reconhecidamente o agente causal mais comum de meningite em crianças e que apresenta a surdez como principal morbidade correlata. No Brasil, entretanto, só recentemente tais vacinas se tornaram obrigatórias e as campanhas de vacinação atingiram patamares de adesão mais elevados.

O impacto do uso indiscriminado e da má utilização da antibioticoterapia também pode ser atenuado, através da melhoria das práticas médicas, devendo também se atentar para o manuseio, por muitas vezes equivocado, das otites de repetição. Medidas educacionais e informativas gerais voltadas para a população como um todo, tais como educação para o trânsito e cuidado com acidentes domésticos, atuarão sobre os casos de surdez devidos a traumatismo crânio encefálico ou à parada cardiorrespiratória.

No grupo de alunos do INES, com déficit auditivo por causas genéticas, encontramos casos de síndrome de Waardenburg, que é a causa sindrômica habitualmente mais encontrada, assim como casos de doença de Usher, Sahetre-Chotzen, entre outros. A maior dificuldade em estabelecer um diagnóstico etiológico genético específico era esperada, pois $70 \%$ dos pacientes com surdez genética não apresentam quaisquer outros sinais clínicos associados, passíveis de serem utilizados como critério diagnóstico, e embora a surdez genética de caráter sindrômico seja conhecida desde o século XIX, os primeiros genes associados à surdez não sindrômica só foram descobertos a partir de 1996, estando ainda longe da nossa realidade a possibilidade de exames biomoleculares precisos que corroborem o processo de diagnose e ofereçam diretrizes mais seguras para a realização do aconselhamento genético.

Nesse sentido, a maior parte de nossos diagnósticos foi feita através de critérios clínicos. A utilização de exames complementares como tomografias, ressonâncias, erros inatos do metabolismo, audiometrias, cariótipos, dentre outros, embora indicada para a avaliação clínica da amostra, encareceria muito o projeto e possivelmente não traria tantos benefícios, como já avaliado pelo estudo de Reardon 39 para a surdez.

Uma série de dificuldades para a conclusão dos protocolos de pesquisa foi encontrada pela equipe. Muitas das informações foram obtidas a partir da mãe, confiando-se na memória de eventos que por vezes ocorreram há muitos anos. Entre as diversas intercorrências perinatais descritas pelas famílias, a não confiabilidade das informações nos levou apenas a considerar fatos inequivocamente marcantes, como, por exemplo, internação no berçário logo após o nascimento. Mesmo nestas circunstâncias, a falta de relatórios médicos não nos permitiu uma discussão mais aprofundada a este respeito.

\section{Considerações finais}

Nosso estudo visa aumentar os conhecimentos com relação à população de deficientes auditivos. Tal conhecimento é fundamental para traçar ações de intervenção no problema em seus vários níveis: primariamente na prevenção, via campanhas de esclarecimento e aconselhamento genético; no nível secundário, na identificação precoce e, terciariamente, em termos de reabilitação no tratamento de condições associadas objetivando a melhoria do prognóstico.

Torna-se necessário alertar para o fato de que a surdez é vinte vezes mais prevalente do que a fenilcetonúria, uma condição que os programas de triagem neonatais brasileiros estão correntemente rastreando. Os avanços tecnológicos para screening da perda auditiva em recém-natos são relativamente baratos e envolvem, basicamente, o método de emissões otoacústicas, indolor, que leva cerca de cinco minutos para ser feito, podendo, inclusive, ser realizado com a criança dormindo 2 .

Com relação ao aconselhamento genético, há que se ter em mente seu propósito de garantir que pacientes/pais compreendam os achados e limitações inerentes a qualquer avaliação genética. Prestar serviços genéticos eficientes requer que os profissionais sejam sensíveis às diferenças culturais e às necessidades psicossociais dos pacientes, assim como estarem permanentemente 
alertas para o processo social da comunicação e seus entraves. Pelo menos $10 \%$ das crianças surdas nascem de pais também surdos, cuja concepção que têm de si mesmos pode ser a de culturalmente serem deficientes auditivos. Nesse caso, a compreensão da diferença de estilos de pensamento deve ser sempre considerada, assim como o princípio da autonomia, o que não significa o abandono da ênfase nas iniciativas de gestão pública. Riscos sociais também precisam ser pensados. Apesar do avanço na legislação e da obrigação da confidencialidade das informações, a discriminação e até a impossibilidade de obtenção de seguro saúde são preocupações comuns às pessoas que procuram o aconselhamento.

Há a necessidade premente de novos estudos a serem feitos com a parcela considerada idiopática, o que, provavelmente, levará a ampliação do reconhecimento dos casos de surdez de etiologia genética, e conseqüentemente ao estabelecimento, dentro dos princípios constituintes do Sistema Único de Saúde (SUS), da assistência integral a esse grupo populacional, em centros de referência, no nível regional.

As crianças identificadas com perda de auditiva podem se beneficiar com os meios de amplificação, a partir dos quatro de anos de idade. Com apropriada intervenção precoce poderão freqüentar escolas regulares de ensino fundamental, médio e universitário. Comunicado da Hearing Loss Organization 2 aponta que estudos recentes concluíram que crianças com deficiência auditiva congênita, diagnosticada antes dos seis meses de idade, demonstram compreensão de linguagem e leitura superior àquelas identificadas posteriormente.

Esperamos que este estudo, ainda em evolução, possa servir como uma chamada de atenção que leve à necessária preocupação com esta população. O melhor conhecimento de suas características tornará possível implementar estratégias de intervenção impulsionadoras de sua interação na sociedade, começando por instituir, na prática, protocolos para a detecção precoce da perda auditiva.

\section{Resumo}

O estudo das deficiências múltiplas em geral e da surdez em especial é escasso em nosso país. Os pesquisadores iniciaram estudo pioneiro desde 1992, avaliando alunos da educação especial das redes governamentais de ensino do Estado, estando atualmente focados na surdez. Avaliamos 232 alunos com idade variando de 1 a 39 anos, com média de 10,9 anos. O sexo masculino prevaleceu na amostra. A consangüinidade ocorreu 7,6\% e a história familiar de surdez em 19\% dos casos. Observamos $33 \%$ de intercorrências gestacionais. O parto normal foi utilizado em 59\% de nossos casos, sendo a termo em $75 \%$. As intercorrências neonatais estiveram presentes em $35 \%$ das vezes. As causas ambientais foram responsáveis por $58,5 \%$ da amostra, as causas genéticas por $20,7 \%$ dos casos. Sendo o restante considerado idiopático. Nossos achados corroboram os dados da literatura. Acreditamos que este estudo possa servir como o início de uma preocupação maior com esta população, e que através do melhor conhecimento de suas características seja possivel implementar estratégias de intervenção facilitando a sua interação produtiva na sociedade.

Pessoas com Insuficiência Auditiva; Estudos Transversais; Educação Especial

\section{Colaboradores}

E. J. C. Silva realizou a coleta, o armazenamento e a aná lise dos dados, assim como participou na elaboração e redação do artigo. J. C. Llerena Jr. concebeu o desenho da pesquisa, supervisionou toda a sua excussão e participou na revisão e redação do artigo. M. H. C. A. Cardoso participou da pesquisa bibliográfica, da revisão dos dados e da elaboração e redação do artigo. 


\section{Referências}

1. Organização Mundial da Saúde. Retardamento mental: enfrentando o desafio. Washington DC: Organização Mundial da Saúde; 2000.

2. Help for Hearing Loss. Medical aspects of hearing loss. http://www.hearinglossweb.com/Medical. Htm\#deaf (acessado em 09/Ago/2004).

3. Hearing Loss Organization. Facts on hearing loss. http://www.shhh.org/html/hearing_loss_fact_ sheets.html (acessado em 13/Abr/2004).

4. Marazita ML, Ploughmam LM, Rawlings B, Remington E, Arnos KS, Nance WE, et al. Genetic epidemiological studies of early-onset deafness in the U.S. school-age population. Am J Med Genet 1993; 46:486-91.

5. The Rockefeller University. The Rockefeller University study on the genetics of non syndrome hearing loss. http://linkage.rockefeller.edu/nshl (acessado em $11 / \mathrm{Jul} / 2004$ ).

6. Silva EJC. A educação especial como referencial de estudo das deficiências múltiplas [Dissertação de Mestrado]. Rio de Janeiro: Universidade Federal do Rio de Janeiro; 1997.

7. Llerena Jr. JC, Silva EJC, Horovitz DDG, Correia P, Mascarenhas E, Camacho L. Investigação do retardo mental e doenças genéticas a partir de um estudo transversal em escolas do Estado do Rio de Janeiro. Inf Epidemiol SUS 2000; 3:77-83.

8. Silva EJC. Investigação clínico-epidemiológica em um modelo de educação inclusiva visando ao estudo dos transtornos do desenvolvimento [Tese de Doutorado]. Rio de Janeiro: Instituto Fernandes Figueira, Fundação Oswaldo Cruz; 2002.

9. Lefrève $A B$. Exame neurológico evolutivo. In: Diament A, Cypel S, organizadores. Neurologia infantil. Rio de Janeiro: Editora Atheneu; 1996. p. 99110.

10. Jones KL. Smith's reconizable patterns of human malformation. Philadelphia: W. B. Saunders Company; 1997.

11. Castilla EE, Oriolli LM. El estudio colaborativo latinoamericano de malformaciones congenitas: ECLAMC/Monitor. Interciência 1983; 8:271-8.

12. Gorlin RJ, Toriello HV, Cohen Jr. MM. Epidemiology, etiology, and genetic patterns. Hereditary hearing loss and its syndromes. Oxford: Oxford Monographs on Medical Genetics; 1995.

13. Rodrigues $\mathrm{MH}$, Noronha $\mathrm{MH}$. O deficiente da audição e a educação especial. Rio de Janeiro: Editora José Olympio; 1994.

14. Parving A, Hauch AM, Christensen B. Hearing loss in children-epidemiology, age at identification and causes through 30 years. Ugeskr Laeger 2003; 165:574-9.

15. Joint Committee on Infant Hearing. Year 2000 position statement: principles and guidelines for early hearing detection and intervention programs. Am J Audiol 2000; 9:9-29.

16. Glover NM, Glover JG. Ethical and legal issues regarding selective abortion of fetuses with Down syndrome. Ment Retard 1996; 7:207-14.

17. Kollee LA, van der Heide A, de Leeuw R, van der Maas PJ, van der Wal G. End-of-life decisions in neonates. Semin Perinatol 1999; 23:234-41.
18. Kunen S, Overstreet S, Salles C. Concurrent validity study of the Slosson Intelligence Test-Revised in mental retardation testing. Ment Retard 1996; 34:380-6.

19. Pinkerton F, Watson DR, McClelland RJ. A neurophysiological study of children with reading, writing and spelling difficulties. Dev Med Child Neurol 1989; 31:569-81.

20. Curran AL, Sharples PM, White C, Knapp M. Time costs of caring for children with severe disabilities compared with caring for children without disabilities. Dev Med Child Neurol 2001; 43:529-33.

21. Dinenssen SJ, Greisen G. Quality of life in young adults with very low birth weight. Arch Dis Child Fetal Neonatal Ed 2001; 85:165-9.

22. Doron MW, Vaness-Meehan KA, Margolis LH, Holoman EM, Stiles AD. Delivery room ressucitation decisions for extremely premature infants. Pediatrics 1998; 102:574-82.

23. Law M, Russell D, Pollock N, Walter S, King G. A comparison of intensive neurodevelopmental therapy plus casting and a regular ocupational therapy program for children. Dev Med Child Neurol 1997; 39:664-70.

24. Levene MI, Evans DJ, Mason S, Brown J. An international network for evaluating neuroprotective thrapy after severe birth asphyxia. Semin Perinatol 1999; 23:226-33.

25. McCabe L, McCabe ERB. Postgenomic medicine. Presymptomatic testing for prediction and prevention. Clin Perinatol 2001; 28:425-34.

26. Instituto Brasileiro de Geografia e Estatística. Dados sobre condições perinatais do censo de 2000. http://www.ibge.gov.br (acessado em 20/ Abr/2004).

27. Cans C. Surveillance of cerebral palsy in Europe: collaboration of cerebral palsy surveys and registers. Dev Med Child Neurol 2000; 42:816-24.

28. Williams K, Alberman E. Survival in cerebral palsy: the role of severity and diagnostic labels. Dev Med Child Neurol 1998; 40:376-9.

29. Fellick JM, Sills JA, Marzouk O, Hart CA, Cooke RW, Thomson APJ. Neurodevelopmental outcome in meningococal disease: a case-control study. Arch Dis Child 2001; 85:6-11.

30. Finnstrom O, Olausson PO, Serenius F, Svenningsen N, Tunnel R, Wesstrom G. Neurosensory outcome and growth at three years in extremely low birthweight infants: follow-up results from Swedish national prospectively study. Acta Paediatr 1998; 87:1055-60.

31. Jacinto SJ, Korrthals MG, Ferreira JA. Predicting outcome in hipoxic-ischemic Brain injury. Pediatr Clin North Am 2001; 48:647-60.

32. Ronem GM, Rosenbaum P, Streiner DL. Outcome measures in pediatric neurology: Why do we need them? J Child Neurol 2000; 15:775-8

33. First LR, Palfrey JS. The infant or young child with developmental delay. N Engl J Med 1994; 330: 478-83.

34. Ruos K, Lovbad K, Scrooth G, Fusch C. Brain developmentt (sulci and gyri) as assessed by early postnatal MR imaging in preterm and term newborn infants. Neuropediatrics 2001; 32:69-74. 
35. Vakama AM, Tolonem EU, Pakko ELE, Koivisto ME. Brainstem size and function at term age in relation to later neurosensory disability in high risk preterm infants. Acta Paediatr 2001; 90:909-15.

36. Scher MS. Neurophysiological assessment of brain function and maturation: a measure of brain adaptation in high risk infants. Pediatr Neurol 1997; 16:191-8.

37. Larson A. Neonatal screening for metabolic, endocrine, infectious, and genetic disorders. Clin Perinatol 2001; 28:312-21.
38. Papavasiliou AS, Bazigou H, Paraskevoulakos E, Kotsalis C. Neurometabolic testing in developmental delay. J Child Neurol 2000; 15:620-2.

39. Reardon W. Genetic deafness. J Med Genet 1992; 29:521-6.

Recebido em 08/Mar/2005

Versão final reapresentada em 12/Mai/2006

Aprovado em 10/Jul/2006 